\title{
Dynamical origin of nonstrange baryon resonances
}

\author{
K. P. Khemchandani* \\ Instituto de Física, Universidade de São Paulo, C.P 66318, 05314-970 \\ São Paulo, SP, Brazil \\ E-mail: kanchaneif.usp.br
}

\section{A. Martínez Torres}

Instituto de Física, Universidade de São Paulo, C.P 66318, 05314-970

São Paulo, SP, Brazil

E-mail: amartinedif.usp.br

\section{H. Nagahiro}

Department of Physics, Nara Women's University, Nara 630-8506, Japan Research Center for Nuclear Physics (RCNP), Mihogaoka 10-1, Ibaraki 567-0047, Japan

and

Research Center for Nuclear Physics (RCNP), Mihogaoka 10-1, Ibaraki 567-0047, Japan.

E-mail: nagahiro@rcnp.osaka-u.ac.jp

\section{A. Hosaka}

Research Center for Nuclear Physics (RCNP), Mihogaoka 10-1, Ibaraki 567-0047, Japan

E-mail: hosaka@rcnp.osaka-u.ac.jp

In this talk we discuss about our recent work on nonstrange meson-baryon systems. Our objective is to study coupled channel meson-baryon interaction by considering the vector and pseudoscalar mesons and look for dynamically generated baryon resonances in such systems. For this we solve the Bethe-Salpeter equation with kernels obtained from chiral and hidden local symmetry Lagrangians. For the interactions between pseudoscalar mesons and baryons we rely on the Weinberg-Tomozawa theorem. When dealing with vector mesons, we calculate the $s^{-}, t_{-}, u$ channel diagrams and a contact term. Our study resulted in finding of resonances which can be related to $N^{*}(1535), N^{*}(1650), N^{*}(1895)$ and $\Delta(1620)$. We find that these resonances can be, at least partly, interpreted as dynamically generated resonances.

XV International Conference on Hadron Spectroscopy-Hadron 2013

4-8 November 2013

Nara, Japan

\footnotetext{
*Speaker.
} 


\section{Introduction}

With the motivation to study the properties of the baryon resonances and their coupling to vector and pseudoscalar mesons, we have investigated interaction of these mesons with octet baryons in Refs. $[1,2,3]$. In these works the vector meson-baryon (VB) interaction was obtained from the Lagrangian based on the hidden local symmetry (HLS) which treats vector mesons as the gauge bosons. Using this Lagrangian we calculated the $s$-, $t$ - and $u$-channel diagrams and a contact interaction which comes directly from the two meson-field part of the Lagrangian, which is related to the anomalous magnetic moment of baryons, and found all the amplitudes to be of similar order [1]. Further, we found that the contact interaction is also important to be considered in order to keep the gauge invariance of the hidden local symmetry. Thus, unlike the case of pseudoscalar meson where one can rely on the Weinberg-Tomozawa theorem, the $t$-channel interaction does not give dominant contribution to the VB interaction. In fact, the VB $t$-channel interaction turns out to be spin independent (as shown in Ref. [4]) while the addition of other diagrams lifts this degeneracy and leads to a spin-isospin dependent amplitude, as could be expected from the interaction of two particles with a similar mass and nonzero spin.

To include the pseudoscalar mesons in our formalism we use the Kroll-Ruderman theorem for the photoproduction of pions and replace the photon by a vector meson which is introduced as the gauge boson of the HLS $[2,3]$. We obtain the pseudoscalar-baryon (PB) amplitudes by using the lowest order chiral Lagrangians and by relying on the Weinberg-Tomozawa theorem.

We have earlier studied strangeness -1 meson baryon systems [2,3] and found some useful information related to $\Lambda(1405), \Lambda(1670), \Lambda(2000), \Sigma(1750), \Sigma(1940), \Sigma(2000)$.

More recently we extended our study to the nonstrange meson-baryon systems [5]. Obtaining reliable information on the properties of nonstrange baryon resonances is important for the low energy nuclear-hadron physics. These resonances play an important role in understanding $N-N$ interaction [6,7], in describing cross sections for the reaction with meson-nucleus final states (for instance, see Refs. $[8,9,10,11])$, in approaching some fundamental issues, like, existence of multiquark states [12], occurrence of OZI violating processes [13], etc. In fact a lot of work has been done to study the $N^{*}$ and $\Delta$ resonances using partial wave analyses or different dynamical coupled channel models $[14,15,16,17,18,19,20,21,22]$. The distinguishing features of our work are the treatment of vector and pseudoscalar mesons in full coupled channel formalism with the interaction kernels obtained from the lowest order Lagrangians based on the HLS and the consideration of diagrams beyond the $t$-channel interaction for the vector meson-baryon systems. We discuss some important findings of our work in the next section.

\section{Theoretical framework and results}

To obtain the vector meson-baryon amplitudes we start with the SU(2) Lagrangian

$$
\mathscr{L}_{\rho N}=-g \bar{\psi}\left\{\gamma_{\mu} \rho^{\mu}+\frac{\kappa_{\rho}}{4 M} \sigma_{\mu \nu} \rho^{\mu v}\right\} \psi,
$$

and require that the nucleon fields $(\psi)$ transform under the hidden local symmetry (HLS) as $\psi \rightarrow$ $h(x) \psi$, where $h(x)$ is an element of the HLS. We find that the condition of the gauge invariance is 
satisfied only when the commutator part of the tensor field $\rho^{\mu v}$,

$$
\rho^{\mu v}=\partial_{\mu} \rho_{v}-\partial_{v} \rho_{\mu}+i g\left[\rho_{\mu}, \rho_{v}\right],
$$

is taken into account. This term gives rise to a contact interaction which, in addition to being important for the gauge invariance, turns out to be comparable, in magnitude, to the $t$-channel amplitude. This Lagrangian has been generalized to SU(3) in Refs. $[1,2,3]$ where amplitudes for different diagrams have been obtained for different VB vertices.

Further, this formalism has been extended to study PB-VB coupled channels. To obtain the PB $\leftrightarrow$ VB amplitudes we start by using the Kroll-Ruderman theorem to write the Lagrangian for the $\gamma N \rightarrow \pi N$ process and replace the $\gamma$ by the $\rho$-meson via the notion of the vector meson dominance. This gives the $\pi N \rightarrow \rho N$ amplitude

$$
\mathscr{L}_{\pi N \rho N}=-i \frac{g g_{A}}{2 f_{\pi}} \bar{N}\left[\pi, \rho^{\mu}\right] \gamma_{\mu} \gamma_{5} N
$$

where $\pi=\vec{\tau} \cdot \pi$ and $\rho=\vec{\tau} \cdot \frac{\rho}{2}$. Generalizing the Lagrangian in Eq. (2.3) for the SU(3) case [2], we get

$$
\mathscr{L}_{P B V B}=\frac{-i g}{2 f_{\pi}}\left(F\left\langle\bar{B} \gamma_{\mu} \gamma_{5}\left[\left[P, V_{\mu}\right], B\right]\right\rangle+D\left\langle\bar{B} \gamma_{\mu} \gamma_{5}\left\{\left[P, V_{\mu}\right], B\right\}\right\rangle\right),
$$

where the trace $\langle\ldots\rangle$ has to be calculated in the flavor space and $F=0.46, D=0.8$ such that $F+D \simeq g_{A}=1.26$. The amplitudes for the different nonstrange $\mathrm{PB} \leftrightarrow \mathrm{VB}$ transitions are given in Ref. [5].

We obtain the remaining amplitudes, which are those for the PB channels, by calculating Weinberg-Tomozawa terms using chiral Lagrangians.

With these kernels we solve the Bethe-Salpeter equation. The loop functions for this are calculated using the dimensional regularization scheme and the required subtraction constants are fixed by fitting the data on the isospin $1 / 2$ and $3 / 2 \pi N$ amplitudes and on the reactions: $\pi^{-} p \rightarrow \eta n$ and $\pi^{-} p \rightarrow K^{0} \Lambda$. The resulting subtraction constants can be found in Ref. [5]. We show the results obtained with these subtraction constants for the isospin $1 / 2$ and $3 / 2 \pi N$ amplitudes, in Fig. 1, as solid and dashed lines, respectively. The figure also shows the experimental data, taken from Ref. [23], on the real (imaginary) part of the $\pi N$ amplitudes, for comparison, as dotted (dashdotted) lines. We should mention here that the calculations have been done for the spin-parity $1 / 2^{-}$configuration. The results on the $3 / 2^{-}$resonances can be found in Ref. [1], which we couple to vector mesons only. In fact it was found in Ref. [24] that the PB-VB coupling is weak in the $3 / 2^{-}$case.

To study the properties of the resonances contributing to the PB-VB coupled channel scattering in the $1 / 2^{-}$partial wave, we search for poles in the complex plane. As a result we find some poles which can be related to $N^{*}(1535), N^{*}(1650), N^{*}(1895)$ and $\Delta(1620)$. The pole which we relate to the $N^{*}(1535)$ resonance is found at $1504-i 55 \mathrm{MeV}$. We also calculate its branching ratios to the $\pi N$ and $\eta N$ channels which turns out to be $43 \%$ and $55 \%$, respectively. These results are in good agreement with the known properties of $N^{*}(1535)$ [25].

In the case of $N^{*}(1650)$, we find a double pole nature for it, with positions $1668-i 28 \mathrm{MeV}$ and $1673-i 67 \mathrm{MeV}$. We find that twin poles unavoidably appear in this energy region while minimizing the $\chi^{2}$ to fit the data. Such a nature of $N^{*}(1650)$ has already been reported in Ref. [23]. 

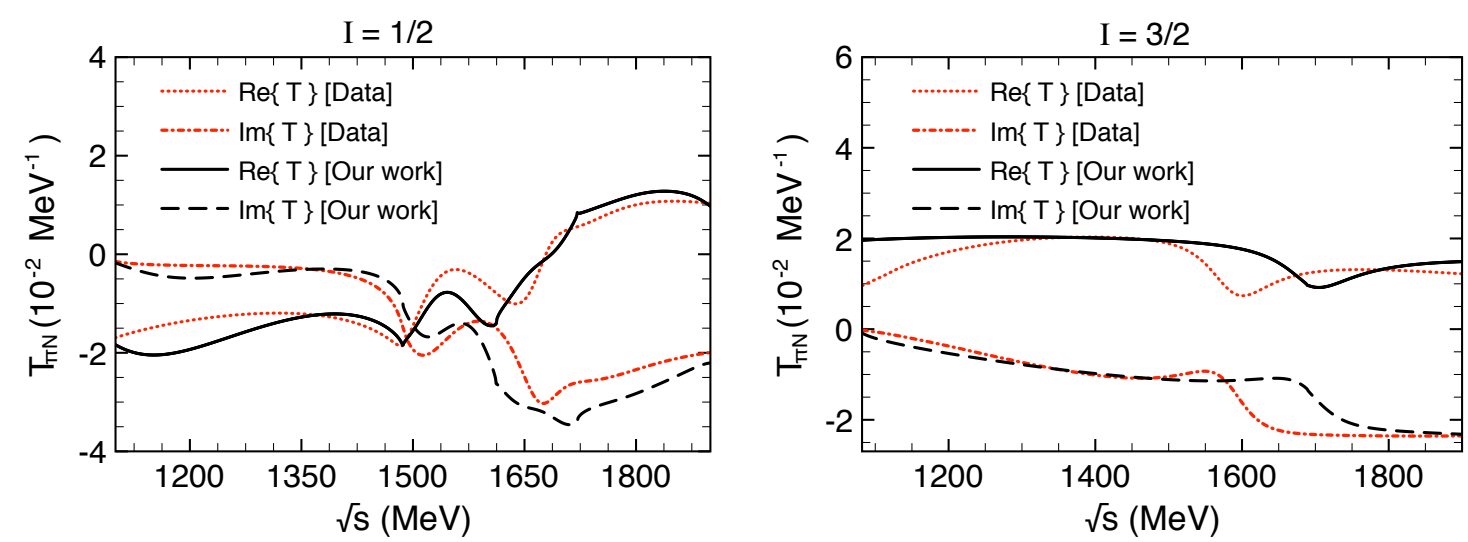

Figure 1: Real and imaginary parts of the isospin 1/2 (left panel) and 3/2 (right panel) amplitudes for the $\pi N$ channel.

Next, we find resonances at $1801-i 96 \mathrm{MeV}$ and $1912-i 54 \mathrm{MeV}$ in the isospin half case. Although they do not show as a double hump in the amplitudes on the real axis due to their overlapping large widths. For example, a bump, and not a double peak like structure, is seen around $1900 \mathrm{MeV}$ in our results on the cross sections for the $\pi^{-} p \rightarrow \eta N$ reaction, which seems to be in good agreement with the experimental data (as shown in Ref. [5]). This indicates that the identification of these resonances in the experimental data can be difficult, and it could be the reason for the large variation on the masses and widths found for $1 / 2^{-}$resonances around $1900 \mathrm{MeV}$ (put together under $N^{*}(1895)$ [25]).

In the case of isospin $3 / 2$ we find a pole at $1689-i 56 \mathrm{MeV}$ which we associate with the well known $\Delta(1620)$ resonance, for which the mass and the width are given in the range of 1600-1660 $\mathrm{MeV}$ and 130-150 MeV, respectively, in Ref. [25]. This shows that our state has a slightly higher mass. Although other properties of $\Delta(1620)$ found in our work seem to be in good agreement with the known ones [25], like a significant branching ratio to $\rho N$ and a smaller one to the $\pi N$ channel in spite of the presence of a larger phase space in the latter case (as also found in Ref. [26]).

Finally, we would like to mention that we have tried to interpret the nature of the resonances found in our work on the basis of Ref. [27]. We find that with the addition of vector mesons to build the coupled channels we seem to move in the direction of understanding the low-lying $N^{*}$ and $\Delta$ resonances as dynamically generated states.

\section{References}

[1] K. P. Khemchandani, H. Kaneko, H. Nagahiro, A. Hosaka, Phys. Rev. D83, 114041 (2011).

[2] K. P. Khemchandani, A. Martinez Torres, H. Kaneko, H. Nagahiro and A. Hosaka, Phys. Rev. D 84, 094018 (2011).

[3] K. P. Khemchandani, A. Martinez Torres, H. Nagahiro and A. Hosaka, Phys. Rev. D 85, 114020 (2012).

[4] E. Oset and A. Ramos, Eur. Phys. J. A 44, 445 (2010). 
[5] K. P. Khemchandani, A. Martinez Torres, H. Nagahiro and A. Hosaka, Phys. Rev. D 88, 114016 (2013); K. P. Khemchandani, A. Martinez Torres, H. Nagahiro and A. Hosaka, arXiv:1310.0067 [nucl-th].

[6] I. Maeda, M. Arima and K. Masutani, Phys. Lett. B 474, 255 (2000).

[7] S. Furuichi and T. Sokawa, Prog. Theor. Phys. 41, 1504 (1969).

[8] V. Shklyar, H. Lenske, U. Mosel and G. Penner, Phys. Rev. C 71, 055206 (2005) [Erratum-ibid. C 72, 019903 (2005)]; G. Penner and U. Mosel, Phys. Rev. C 66, 055212 (2002).

[9] M. Doring, C. Hanhart, F. Huang, S. Krewald, U. -G. Meissner and D. Ronchen, Nucl. Phys. A 851, 58 (2011); E. J. Garzon, J. J. Xie and E. Oset, Phys. Rev. C 87, 055204 (2013).

[10] K. P. Khemchandani, N. G. Kelkar and B. K. Jain, Nucl. Phys. A 708, 312 (2002); N. J. Upadhyay, K. P. Khemchandani, B. K. Jain and N. G. Kelkar, Phys. Rev. C 75, 054002 (2007).

[11] K. Schonning et al. [CELSIUS/WASA Collaboration], Phys. Rev. C 79, 044002 (2009).

[12] B. Julia-Diaz and D. O. Riska, Nucl. Phys. A 780, 175 (2006) [nucl-th/0609064].

[13] J. -J. Xie, B. -S. Zou and H. -C. Chiang, Phys. Rev. C 77, 015206 (2008); X. Cao, Chin. Phys. C 33, 1381 (2009); M. Doring, E. Oset and B. S. Zou, Phys. Rev. C 78, 025207 (2008) [arXiv:0805.1799 [nucl-th]].

[14] C. Hanhart, Phys. Rept. 397, 155 (2004); O. Krehl, C. Hanhart, S. Krewald and J. Speth, Phys. Rev. C 62, 025207 (2000) [arXiv:nucl-th/9911080].

[15] F. Huang, H. Haberzettl and K. Nakayama, Phys. Rev. C 87, 054004 (2013).

[16] H. Kamano, PoS QNP 2012, 011 (2012).

[17] M. F. M. Lutz and E. E. Kolomeitsev, Nucl. Phys. A 755, 29 (2005); E. E. Kolomeitsev and M. F. M. Lutz, Phys. Lett. B 585, 243 (2004); C. Garcia-Recio, M. F. M. Lutz and J. Nieves, Phys. Lett. B 582, 49 (2004).

[18] J. M. Alarcon, J. M. Camalich and J. A. Oller, PoS ConfinementX , (2012) 122; J. M. Alarcon, J. M. Camalich and J. A. Oller, Annals Phys. 336, 413 (2013).

[19] E. Oset, S. Sarkar, B. X. Sun, M. J. Vicente Vacas, A. Ramos, P. Gonzalez, J. Vijande and A. Martinez Torres et al., Nucl. Phys. A 835, 271 (2010); F. Aceti, L. R. Dai, L. S. Geng, E. Oset and Y. Zhang, arXiv:1301.2554 [hep-ph]; A. Ramos and E. Oset, Phys. Lett. B 727, 287 (2013)

[20] A. Martinez Torres, D. Jido, Phys. Rev. C82, 038202 (2010); A. Martinez Torres, K. P. Khemchandani, E. Oset, Phys. Rev. C79, 065207 (2009); A. Martinez Torres, K. P. Khemchandani, U. -G. Meissner and E. Oset, Eur. Phys. J. A 41, 361 (2009).

[21] C. Garcia-Recio, J. Nieves and L. L. Salcedo, Eur. Phys. J. A 31, 499 (2007); C. Garcia-Recio, J. Nieves and L. L. Salcedo, Phys. Rev. D 74, 034025 (2006).

[22] D. Gamermann, C. Garcia-Recio, J. Nieves and L. L. Salcedo, Phys. Rev. D 84, 056017 (2011).

[23] R. A. Arndt, I. I. Strakovsky, R. L. Workman and M. M. Pavan, Phys. Rev. C 52, 2120 (1995).

[24] E. J. Garzon and E. Oset, Eur. Phys. J. A 48, 5 (2012).

[25] J. Beringer et al. (Particle Data Group), Phys. Rev. D 86, 010001 (2012).

[26] J. -J. Xie and B. -S. Zou, Phys. Lett. B 649, 405 (2007).

[27] T. Hyodo, D. Jido, A. Hosaka, Phys. Rev. C 78, 025203 (2008). 